\title{
Peertechz
}

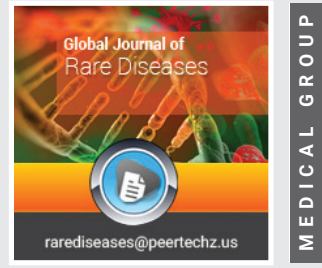

\section{Delayed splenic rupture 4 months following minor blunt abdominal trauma}

\author{
Chatzipetrou Maria, Tzanakis Nickolaos, Giannopoulos \\ George and Kornaropoulos Michail*
}

Department of Surgery, Asklepieio General Hospital Voula, Athens, Greece
Received: 26 April, 2021

Accepted: 12 May, 2021

Published: 13 May, 2021

*Corresponding author: Kornaropoulos Michail, MD, MsC, Department of Surgery, Asklepieio General Hospital Voula, Athens, Greece, Tel: +30 6944517352; E-mail:mikekornaro@gmail.com, bxeir@asklepieio.gr

Keywords: Delayed splenic rupture; Atraumatic splenic rupture; Splenic traumac

https://www.peertechzpublications.com

\section{Check for updates}

\section{Background}

Splenic rupture, no matter what the cause is, is associated with significant mortality. It can present either acutely after trauma, or days to weeks later, a situation known as "delayed splenic rupture (DSR)". On the other hand, splenic rupture without trauma history occurs mainly in a pathologically altered spleen, a rare situation called atraumatic splenic rupture (ASR). Cases of ASR of normal spleen have been reported in literature with various cause as far as trauma is concerned.

Regardless of etiology, management of splenic rupture can be divided into non-operative and operative, depending on the grade of splenic injury and the hemodynamic stability. Nonoperative management (NOM) of splenic trauma has become the common treatment in hemodynamically stable patients with $90 \%$ success. Nevertheless, there should be a concern for complications from NOM, such as hematoma, splenic pseudocyst, sepsis and delayed rupture.

We herein report a rare case of delayed splenic rupture, caused by a minor blunt abdominal trauma 4 months prior presentation. After all the other causes of atraumatic splenic rupture were excluded with thorough examinations, we concluded that our case may be one of the longest reported time intervals of DSR, between splenic injury and delayed rupture.

DSR should be considered in the differential diagnosis of an acute surgical abdomen, irrespectively of time and mechanism of the inciting event.

\section{Case study}

\section{Case presentation}

A 42-year-old Greek male, cruise ship captain, presented in the emergency department complaining for left upper abdominal pain for the last 12 hours. He had no history of fever, vomiting or any bowel symptoms. He reported no recent trauma and his medical history was unremarkable. On arrival, he was fully conscious, with blood pressure 130/70 mm Hg, mildly tachycardic (110/min) and normal oxygen saturation. He could only recall that the pain started right after defecation the previous night, worsening the last two hours. On examination, his abdomen was tender on the left side, without peritoneal signs. Initial blood tests showed Hgb of $12 \mathrm{gm} \%$ and $\mathrm{Hbt}$ of $38 \%$, otherwise within normal range.

\section{Investigation}

Patient underwent a thorough imaging investigation. Chest X-Ray was normal, but abdominal ultrasonography (US) showed moderate free fluid around liver, spleen and pelvis. Abdominal computerized scan (CT) revealed splenic rupture with iv contrast extravasation along the superior pole (Figures $1,2)$.

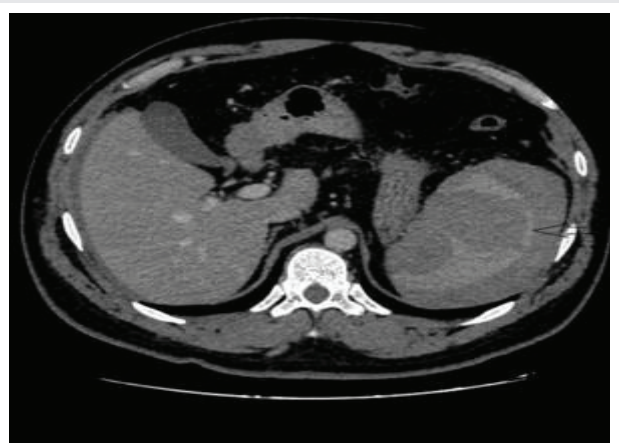

Figure 1: Contrast-enhanced CT portal venous phase, axial image showing multiple laceration, intraparenchymal hematoma at the upper pole of the spleen (arrow) and massive hemoperitoneum.c 


\section{Treatment}

Laparotomy was decided because of clinical deterioration (110p/min, BP 90/50). Exploratory laparotomy revealed hemoperitoneum due to a large ruptured subcapsular hematoma in the superior splenic pole. Splenectomy was performed and no other abnormalities were noted (Figure 3).

\section{Postoperative course}

Postoperative course was uneventful and he was discharged on the postoperative day 5 . Considering his occupation and exclusion of trauma, a thorough infectious disease workup was done. Nonetheless, blood cultures and serologies for viral diseases (CMV, EBV, HIV, Hep) were negative. Blood smear also showed no atypical lymphocytes or any other abnormalities.

The removed spleen measured $15 \times 11 \times 5 \mathrm{~cm}$ and weighed 360 gr. Histology described superior pole rupture with areas of congestion and hemorrhage, apparently of traumatic nature.

Prior to his discharge, patient could eventually recall a trivial abdominal injury on the ship, four months ago. He described it more as pressure of left hypochondrium bending on a hard surface rather than trauma. The pain lasted for 3 days, but he did not seek any medical advice at that time.

\section{Discussion}

Splenic rupture is associated with significant mortality. It can present either acutely after trauma, or days to weeks later,

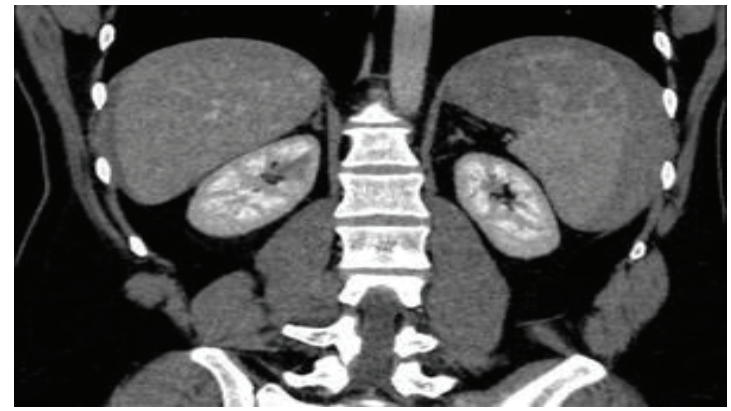

Figure 2: Contrast-enhanced CT portal venous phase coronal reformatted image showing multiple laceration (white arrow), intraparenchymal hematoma at the upper pole of the spleen (black arrow).

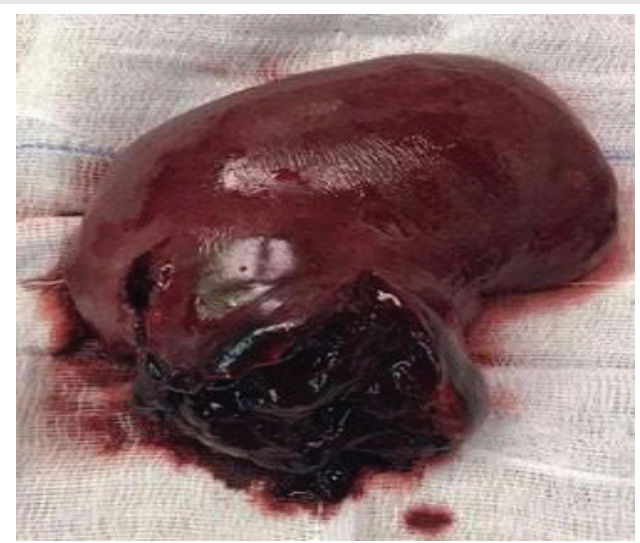

Figure 3: Large parenchymal hematoma in the superior pole of the spleen. a situation known as "delayed splenic rupture (DSR)". DSR is defined as the rupture of the spleen 48 hours after injury and was first described by Baudet in 1902. He also described an asymptomatic period between injury and rupture, known as the "latent period of Baudet" [1].

Various potential mechanisms for DSR have been described. Formation of subcapsular hematoma, temporarily tamponaded by surrounding organs, only to rupture into the abdominal cavity either spontaneously or after a second trauma, seems to be the most common cause. DSR may also arise from splenic parenchymal pseudoaneurysm formation, where a breach in the vascular wall leads to an extravascular hematoma that eventually ruptures, causing hemorrhage. Another possible cause of DSR is the bleeding of internal splenic pseudocyst or a clot disruption [2]. Interestingly, Shinkai et al, reported a case of a subcapsular tear during defecation, due to splenocolic ligament stretching. [3] Cases of splenic injury after colonoscopy, most often caused by traction on the splenocolic ligament or excessive manipulation during the procedure have been reported in the literature [4] Similarly, sneezing, coughing or the distention of the stomach after eating a heavy meal in a combination of rising of intra-abdominal pressure have also been associated with DSR [5].

Unlike traumatic splenic rupture, splenic rupture without injury is extremely rare. Atraumatic splenic rupture (ASR) that occurs in a pathologically altered spleen is called 'pathologic atraumatic splenic rupture', whereas ASR in a histologically proven normal spleen is called "atraumatic idiopathic splenic rupture" $[6,7]$.

The causes of ASR vary and can be classified into six main categories in order of frequency as follows: neoplastic (leukemia, lymphoma), infectious (CMV, EBV, HIV, Hep), hematological (Idiopathic Thrombocytopenic purpura, Hemophilia), inflammatory (pancreatitis, amyloidosis), iatrogenic (drug and treatment related) and primary splenic causes (splenic cyst) [8].

Regardless of etiology, management of splenic rupture can be divided into non operative and operative, depending on the degree of splenic injury as described by the AAST classification and the hemodynamic stability. Non-operative management (conservative management and interventional radiology) has become the standard of care for hemodynamically stable patients in Grades I to III, with $90 \%$ success, whereas injuries of Grade IV and V injuries, with evidence of extravasation and hemodynamically unstable patients, require operative management [9].

Non-operative Management (NOM) has been promoted mainly due to Overwhelming Post Splenectomy Infections (OPSI), a serious complication of splenectomy, reported in $0.5 \%$ of traumatic splenectomies and up to $20 \%$ of nontraumatic causes for splenectomy. OPSI manifests usually in the first two years after surgery, but results in a lifetime risk of infections and carries a mortality of $50-80 \%$ [10]. Lower hospital costs, avoidance of intra-abdominal complications and the maintenance of the immunological function of spleen are the advantages of NOM over splenectomy. 
On the other hand, despite the high rates of success with NOM in patients with low-grade splenic injuries, there should be a concern that current practice may develop increased morbidity and mortality in patients presenting with complications from NOM, such as hematoma, splenic pseudocyst, sepsis and delayed rupture, the most sever one.

In our reported case, after excluding all the possible causes of ARS, by serology, hematology examinations and after the pathology report did not reveal histopathological changes of spleen, we concluded that this is a very rare case of reported DSR. The initial blunt abdominal trauma of our patient while working on the ship probably caused hematoma to the parenchyma but did not injure the capsule. Four months later, after defecation, a tear in this subcapsular hematoma resulted in rupture of the spleen and hemoperitoneum. A possible mechanism for the rupture is the stretching of the splenocolic ligament after defecation.

Taking into account that not every patient with blunt abdominal trauma presents for assessment to the emergency department and that CT abdomen is not performed for every trauma patient, it is possible that cases with spontaneous atraumatic splenic rupture are actually misdiagnosed DSR.

Detailed history of any recent abdominal blunt injury, regardless time and mechanism of the inciting event or a history of NOM for past splenic trauma seems to be important as it could lead to prompt diagnosis of DSR.

\section{Learning points}

Splenic rupture, whatever the cause, is associated with significant mortality. It can present either acutely after trauma, or days to weeks, a situation known as delayed splenic rupture.

Atraumatic splenic rupture, an extremely rare situation, occurs mainly in a pathologically altered spleen and is called 'pathologic atraumatic splenic rupture', whereas in a histologically proven normal spleen is called "atraumatic idiopathic splenic rupture".

Cases of atraumatic idiopathic splenic rupture might actually be misdiagnosed delayed splenic rupture.

Detailed history of any recent abdominal blunt injury, regardless time and mechanism of the inciting event or a history of NOM for past splenic trauma seems to be important as it could lead to prompt diagnosis of DSR.

\section{References}

1. Nagarsheth KH (2015) Delayed Splenic Rupture. In P. J. Papadakos \& M. L. Gestring (Eds.), Encyclopedia of Trauma Care. 434-435. Link: https://bit.ly/3uHpsxu

2. Freiwald S (2010) Late-Presenting Complications After Splenic Trauma. Perm J 14: 41-44. Link: https://bit.ly/2SBCrm2
3. Shinkai T, Ono K, Masumoto K, Urita Y, Gotoh C (2018) A rare mechanism of delayed splenic rupture following the nonoperative management of blunt splenic injury in a child. Surg Case Rep 4: 75. Link: https://bit.ly/3hsfBHQ

4. Enofe I, Burch J, Yam J, Rai M (2020) latrogenic Severe Splenic Injury after Colonoscopy. Case Reports in Gastrointestinal Medicine 2020: 8824720. Link: https://bit.ly/3y7ZaGA

5. Kodikaraa S (2009) Mechanisms of delayed splenic rupture: A new hypothesis. Legal Medicine 11: S515-S517. Link: https://bit.ly/3fgolsH

6. Orloff MJ, Peskin GW (1958) Spontaneous rupture of the normal spleen; a surgical enigma. International Abstracts of Surgery 106: 1-11.

7. Renzulli P, Hostettler A, Schoepfer AM, Gloor B, Candinas D (2009) Systematic review of atraumatic splenic rupture. British Journal of Surgery 96: 11141121. Link: https://bit.ly/3uJmPLv

8. Lam GY, Chan AK, Powis JE (2014) Possible infectious causes of spontaneous splenic rupture: A case report. Journal of Medical Case Reports 8: 396. Link: https://bit.ly/3obJz4r

9. Zarzaur BL, Kozar R, Myers JG, Claridge JA, Scalea TM, et al. (2015) The splenic injury outcomes trial: An American Association for the Surgery of Trauma multi-institutional study. Journal of Trauma and Acute Care Surgery 79: 335-342. Link: https://bit.ly/2R6xfXc

10. Roy P, Mukherjee R, Parik M (2018) Splenic trauma in the twenty-first century: Changing trends in management. Annals of The Royal College of Surgeons of England 100: 650-656. Link: https://bit.ly/3y86Mc6

Discover a bigger Impact and Visibility of your article publication with Peertechz Publications

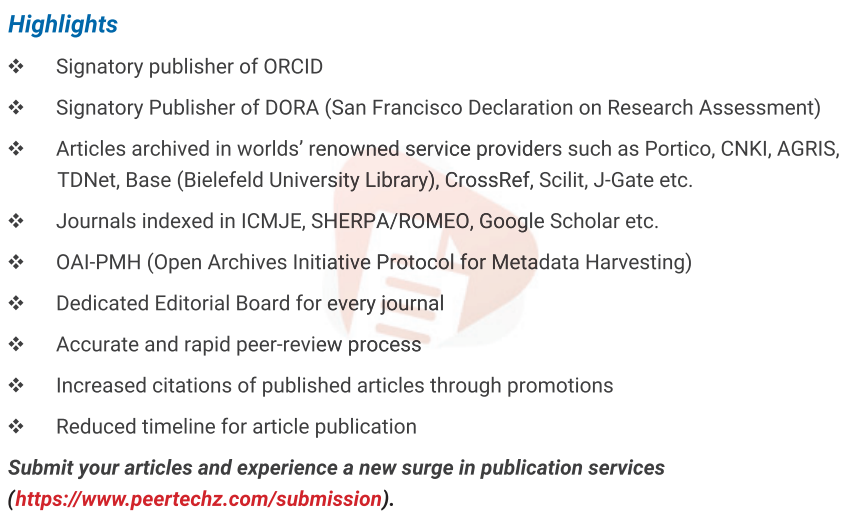

Copyright: @ 2021 Maria C, et al. This is an open-access article distributed under the terms of the Creative Commons Attribution License, which permits unrestricted use distribution, and reproduction in any medium, provided the original author and source are credited. 